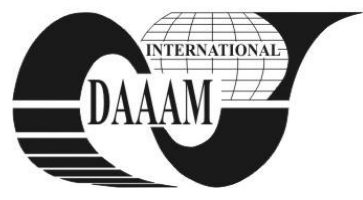

\title{
IN-PROCESS MONITORING OF CUTTING STATES ON CNC TURNING USING SENSOR FUSION VIA NEURAL NETWORK
}

\author{
TANGJITSITCHAROEN, S[omkiat] \& RUNGRUANG, C[hannarong]
}

\begin{abstract}
The aim of this research is to propose and develop the in-process monitoring system of the cutting states of chip and chatter for the carbon steel (S45C) in CNC turning process by utilizing the sensor fusion which are the force sensor, the sound sensor, the accelerometer sensor and the acoustic emission sensor. Their signals have been integrated via the neural network with the perceptron technique to detect the cutting states which are the continuous chip, the broken chip and the chatter occurred. The experimentally obtained results showed that the in-process monitoring system proposed and developed in this research can be effectively used to identify the chip breaking and the chatter with the high accuracy and reliability.
\end{abstract}

Key words: turning, chip, chatter, sensor fusion, neural network

\section{INTRODUCTION}

In turning process, the cutting states of chip and chatter affect the machined surface finish, the accuracy of the work piece, the production cost and the production time. The cutting states of chip formation and chatter are affected by the cutting speed, the feed rate, and the depth of cut. Normally, the broken chip is obtained by reducing the cutting speed, increasing the depth of cut and the feed rate. The chatter is generally avoided by decreasing the depth of cut, the feed rate, and the cutting speed to increase the cutting stability (Somkiat \& Moriwaki, 2008).

Extensive research efforts have been spent to develop the monitoring system. It is already known that the power spectrum densities of three components of the cutting force are utilized to identify the continuous chip, the broken chip and the chatter with the highly reliable performance (Somkiat \& Moriwaki, 2008). The standard deviation, the skewness, the kurtosis, the counted number and the power spectrum ratio of the acoustic emission signals are applied to identify the chip form via the neural network. The artificial neural network has been employed by many researchers in field of manufacturing (Davim et al., 2008; Axinte, 2006; Dong et al., 2006).

The aim of this research is to propose and develop the inprocess monitoring system to identify the cutting states of chip formation and chatter for the carbon steel (S45C) in CNC turning process by utilizing the sensor fusion, which are the force sensor, the sound sensor, the accelerometer sensor and the acoustic emission sensor via the neural network using the perceptron technique.

\section{IN-PROCESS MONITORING OF CUTTING STATES}

2.1 Relations of cutting force, sound, acceleration, acoustic emission, and cutting states

When the chips are broken, the power spectrum densities of three dynamic cutting forces are relatively large (Somkiat, 2008). The standard deviation of the acoustic emission signal becomes larger because the chip breakage is the source of the signal and generates the transient signal. Therefore, the standard deviation of acoustic emission signal is adopted here to identify the broken chip. However, the power spectrum densities of both sound and acceleration signals are also expected to be higher when the broken chip occurs.

When the chatter appears, the power spectrum densities of three dynamic cutting forces become larger at the natural frequency of the system (Somkiat, 2008). In the same way, the power spectrum densities of both sound and acceleration signals are also expected to be higher at the same frequency.

Due to the above mentions, the method is developed to calculate the energy of any signals called the energy spectral density (ESD) from the dynamometer, the sound sensor and the acceleration sensor. It can be calculated by integrating the power spectrum densities of the signals from each sensor over the interested frequency range based on the Rayleigh's energy theorem in order to obtain the new parameters to identify the cutting states of chip formation and chatter via the neural network using the perceptron architecture.

2.2 Monitoring with perception architecture neural network

The perceptron architecture has been created in many variation. One of the simplest is a single-layer network, whose weights and biases can be trained to produce a correct target with the corresponding inputs. The training technique used is called the perceptron learning rule. The perceptron is especially suited for simple problems in the pattern classification because it is fast and reliable networks for the problem that it can solve. In case of the cutting states of chip and chatter, the neural network with the perceptron architecture is used to identify the continuous chip, the broken chip and the chatter as shown in Fig. 1.

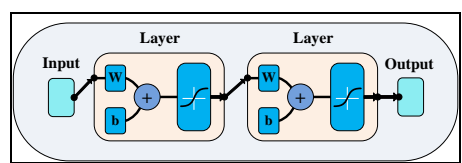

Fig. 1. The structure of the neural network with the perceptron architecture

\section{EXPERIMENTAL EQUIPMENT AND CONDITIONS}

The tool dynamometer is installed on the tool turret of the $\mathrm{CNC}$ turning machine to measure the cutting forces during the cutting process. The accelerometer sensor and the acoustic emission sensor are mounted on the tool shank which is placed on the tool dynamometer. The sound sensor is attached in the CNC turning machine. All sensors are calibrated in advance, hence the signals obtained from all sensors are reliable to identify the cutting states.

The cutting tests are performed by using the coated carbide tool which is popularly used for the turning steel family. The major cutting conditions can be shown in Tab. 1 . 


\begin{tabular}{|l|l|}
\hline Cutting Condition & Dry cutting \\
\hline Cutting tool & Coated carbide tool \\
\hline Tool geometry & DNMG 150604 FN \\
\hline Workpiece & S45C Carbon steel \\
\hline Cutting speed $(\mathrm{m} / \mathrm{min})$ & $100,150,200,250,300$ \\
\hline Feed rate $(\mathrm{mm} / \mathrm{rev})$ & $0.10,0.15,0.20,0.20,0.30$ \\
\hline Depth of cut $(\mathrm{mm})$ & $0.25,0.50,0.75,1.00,1.50,1.75,2.00$ \\
\hline
\end{tabular}

Tab. 1. Major cutting conditions

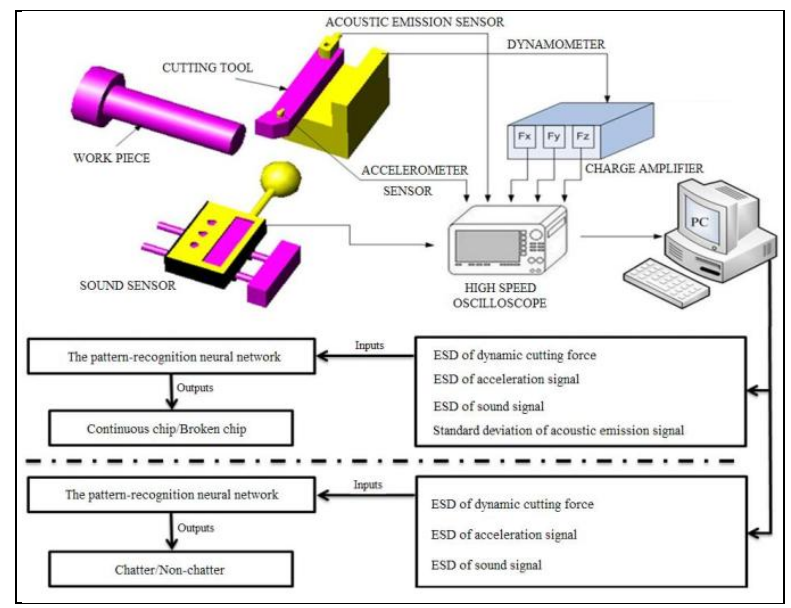

Fig. 2. Illustration of the cutting state identification

The sampling rates are $2 \mathrm{MHz}$ for the acoustic emission sensor and $100 \mathrm{kHz}$ for the accelerometer sensor, the sound sensor and the tool dynamometer. The signal of the cutting force, the acceleration and the sound, are amplified and lowpass filtered with the cut-off frequency of $50 \mathrm{kHz}$ whereas the acoustic emission signal is amplified and low-pass filtered at 1 $\mathrm{MHz}$ prior to digitization and Fast Fourier Transform calculation within PC as shown in Fig. 2.

\section{EXPERIMENTAL RESULTS AND DISCUSSIONS}

According to the experimentally obtained results, when the chips are continuous, the energy spectral densities of the cutting force, the acceleration signal in the range of $100-500 \mathrm{~Hz}$, and the sound signal in the range of $2-3 \mathrm{kHz}$ are significantly lower than that of the broken chips as shown in Figs. 3 (a) to (j). Moreover, the standard deviation of the acoustic emission signal becomes larger when the chips are broken which corresponds to the cutting states.

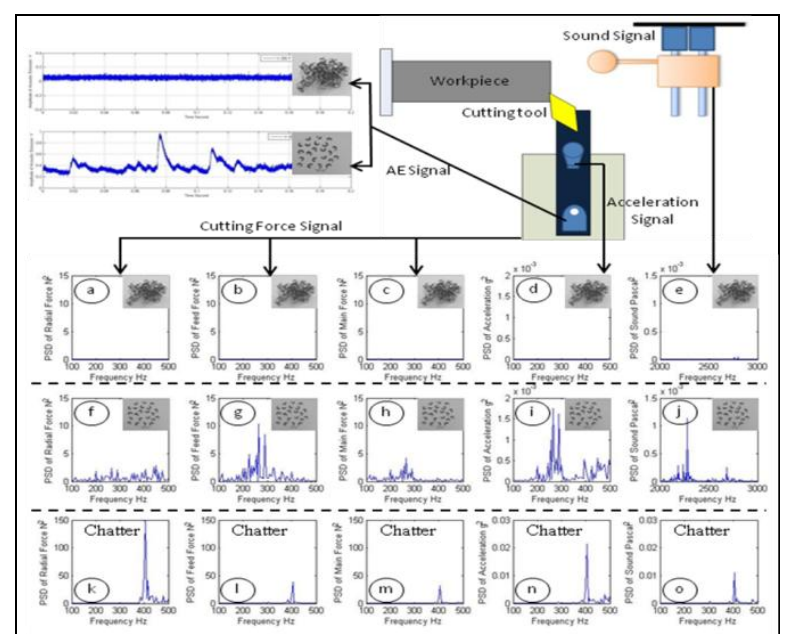

Fig. 3. Illustration of typical examples of experimentally obtained power spectrum densities from four sensors at different cutting states; (a) - (e) continuous chip; (f)-(j) broken chip,(k)-(o)chatter
In order to identify the chip formation, the neural network with the perceptron architecture is applied and trained by using a set of learning data. The inputs of this network consist of the energy spectrum densities of the cutting force, the acceleration signal, and the sound signal as well as the standard deviation of the acoustic emission signal, whereas the output can be used to identify whether the chip is continuous or broken.

The experimental results show that the states of chip can be well identified by the method proposed and developed with the mean absolute percentage error of $4.69 \%$.

It is clearly seen that the chatter frequency is found to be about $400 \mathrm{~Hz}$ as shown in Figs. 3(k) to 3(o). When the chatter occurs, the energy spectral densities of the cutting force, the acceleration signal and the sound signal are prominently large.

The energy spectrum densities of the cutting force, the acceleration signal and the sound signal to identify the chatter are defined as the inputs of the neural network with the perceptron architecture, whereas the output of this network indicates whether the chatter appears or disappears during the cutting. The experimentally obtained results from the proposed and developed method show that the chatter can be efficiently identified with the mean absolute percentage error values less than $8.11 \%$.

\section{CONCLUSIONS}

The experimentally obtained results show that the states of the broken chip, the continuous chip and the chatter can be well identified by the proposed and defined parameters, which are the standard deviation of acoustic emission signal, the energy spectrum densities of three dynamic cutting force components, the sound signal and the acceleration signal.

It has been proved that the proposed method can be effectively used to identify the cutting states via the neural network using the perceptron architecture with the high accuracy and reliability.

\section{ACKNOWLEDGEMENTS}

This research was supported by Faculty of Engineering, Chulalongkorn University from May 2009 to April 2010.

\section{REFERENCES}

Axinte, D. A. (2006). Approach into the use of probabilistic neural networks for automated classification of tool malfunctions in broaching, International Journal of Machine Tools and Manufacture, Vol. 46, No. 12-13, (October 2006), pp. 1445-1448, ISSN: 0890-6955

Davim, J. P.; Gaitonde, V. N. \& Karnik, S. R. (2008). Investigations into the effect of cutting conditions on surface roughness in turning of free machining steel by ANN models, Journal of materials processing technology, Vol. 205, (August 2008), pp. 16-23, ISSN: 0924-0136

Dong, J,; Subrahmanyam, K. V. R.; Wong, Y. S.; Hong, G. S. \& Mohanty, A. R. (2006). Bayesian-inference-based Neural Networks for Tool Wear Estimation, International Journal of Advanced Manufacturing Technology, Vol. 30, (December 2005), pp. 797-807, ISSN: 1433-3015

Somkiat, T. (2008). Development of Intelligent Identification of Cutting States by Spectrum Analysis for CNC Turning, Journal of Advanced Mechanical Design, Systems, and Manufacturing, Vol. 2, No. 3, (October 2007), pp. 366-377, ISSN: 1881-3054

Somkiat, T. \& Moriwaki, T. (2008). Intelligent monitoring and identification of cutting states of chips and chatter on CNC turning machine, Journal of Manufacturing Processes, Vol. 10, No. 1, (January 2008), pp. 40-46, ISSN: 1526-6125 